Research, Society and Development, v. 10, n. 17, e234101724196, 2021

(CC BY 4.0) | ISSN 2525-3409 | DOI: http://dx.doi.org/10.33448/rsd-v10i17.24196

\title{
Benefícios da terapia nutricional enteral em pacientes oncológicos
}

\author{
Benefits of enteral nutrition therapy in cancer patients \\ Beneficios de la terapia de nutrición enteral en pacientes con cáncer
}

Recebido: 01/12/2021 | Revisado: 08/12/2021 | Aceito: 17/12/2021 | Publicado: 27/12/2021

Jardel Alves da Costa

ORCID: https://orcid.org/0000-0002-9844-0770 Universidade Federal do Piauí, Brasil

E-mail: jardelalves@ufpi.edu.br

Nayara Ferreira Ricardo

ORCID: https://orcid.org/0000-0002-6026-9728

Centro Universitário Maurício de Nassau, Brasil

E-mail: nayaratexumo@gmail.com

Renata Rodrigues Costa Fontinele

ORCID: https:/orcid.org/0000-0002-6121-0322 Universidade Federal do Piaú, Brasil

E-mail: renatafontinele25@hotmail.com

Gabrielly Martins de Barros

ORCID: https://orcid.org/0000-0001-9696-424X

Universidade Federal do Piaú, Brasil

E-mail: gabyrhcp00@hotmail.com

Dênaba Luyla Lago Damasceno

ORCID: https://orcid.org/0000-0002-4608-4129

Centro Universitário UNIfacid, Brasil

E-mail:denaba-luyla@hotmail.com

Ana Rafaela Silva Pereira

ORCID: https://orcid.org/0000-0002-9318-2282

Universidade Federal do Piauí, Brasil

E-mail: pereiraars@gmail.com

Diêgo de Oliveira Lima

ORCID: https://orcid.org/0000-0001-8211-9416

Universidade Federal do Piauí, Brasil E-mail: diego.oliveira@ufpi.edu.br

Fernanda Lopes Souza

ORCID: https://orcid.org/0000-0001-8280-9580 Universidade Federal do Piauí, Brasil

E-mail: fernandalopess0102@gmail.com

Ronnyely Suerda Cunha Silva

ORCID: https://orcid.org/0000-0003-3710-7824 Universidade Federal do Piauí, Brasil

E-mail: ronnyelynutricionista@gmail.com

Janaina Morais da Silva

ORCID: https://orcid.org/0000-0001-6089-1181 Universidade Federal do Piauí, Brasil

E-mail: janainamoraisdasilva@gmail.com

Mycaele Aline Santana Sousa

ORCID: https://orcid.org/0000-0001-5654-5905 Universidade Federal do Piauí, Brasil

E-mail: micele29@gmail.com

Cláudia Resende Carneiro

ORCID: https://orcid.org/0000-0002-6593-5283 Universidade Federal do Piauí, Brasil

E-mail: claudiacrc.resende@gmail.com

Cleyde Regina Carvalho de Sousa Gonçalves

ORCID: https://orcid.org/0000-0001-5287-6950 Universidade Federal do Piaú, Brasil

E-mail: cleyde.goncalves@ebserh.gov.br

Lilian Carvalho da Silva

ORCID: https://orcid.org/0000-0001-8654-9915

Centro Universitário Santo Agostinho, Brasil

E-mail: liliancarvalhofenix@hotmail.com 


\section{Resumo}

A pesquisa objetiva realizar revisão literária acerca de estudos que aplicaram na prática clínica condutas nutricionais em nutrição enteral para a terapêutica de pacientes com câncer e relatam os benefícios deste tipo de terapia. Trata-se de uma revisão integrativa da literatura construída a partir da seguinte pergunta norteadora: A terapia nutricional enteral é benéfica para pacientes em tratamento de câncer? A seleção dos estudos foi realizada durante os meses de outubro e novembro de 2021, nas bases de dados: National Library of Medicine (PUBMED), Biblioteca virtual da Elsevier (SCIENCE DIRECT) e Biblioteca Virtual em Saúde (BVS) via portal Periódico Capes. Inicialmente, a estratégia para a busca dos estudos foi composta pela combinação de descritores controlados (Terapia nutricional. Nutrição enteral. Benefícios. Pacientes oncológicos) indexados nos Descritores em Ciências da Saúde (DeCS/MeSH). Após o processo de busca e seleção utilizando os critérios citados na metodologia, foram selecionados 7 artigos. Os estudos analisados e discutidos relatam benefícios significantes desempenhados pela nutrição enteral em pacientes oncológicos, como limitar a perda de peso, fornecer aos pacientes uma ingestão energética adequada, atender às demandas nutricionais aumentadas em decorrência da doença, melhora da função imunológica e função gastrointestinal, recuperação acelerada, redução de complicações pós-operatórias e tempo de hospitalização e melhora dos indicadores nutricionais.

Palavras-chave: Terapia nutricional; Nutrição enteral; Benefícios; Pacientes oncológicos.

\section{Abstract}

The research aims to carry out a literature review on studies that applied nutritional conducts in enteral nutrition in clinical practice for the treatment of cancer patients and report the benefits of this type of therapy. This is an integrative literature review based on the following guiding question: Is enteral nutritional therapy beneficial for patients undergoing cancer treatment? The selection of studies was carried out during the months of October and November 2021, in the following databases: National Library of Medicine (PUBMED), Elsevier Virtual Library (SCIENCE DIRECT) and Virtual Health Library (VHL) via Capes Periodical portal. Initially, the strategy for searching the studies was composed of a combination of controlled descriptors (Nutritional therapy. Enteral nutrition. Benefits. Oncology patients) indexed in the Health Sciences Descriptors (DeCS/MeSH). After the search and selection process using the criteria mentioned in the methodology, 7 articles were selected. The analyzed and discussed studies report significant benefits performed by enteral nutrition in cancer patients, such as limiting weight loss, providing patients with adequate energy intake, meeting increased nutritional demands due to the disease, improvement of immune function and gastrointestinal function, recovery accelerated, reduction of postoperative complications and hospitalization time and improvement of nutritional indicators.

Keywords: Nutrition therapy; Enteral nutrition; Benefits; Cancer patients.

\section{Resumen}

La investigación tiene como objetivo realizar una revisión de la literatura sobre estudios que aplicaron conductas nutricionales en nutrición enteral en la práctica clínica para el tratamiento de pacientes oncológicos y reportar los beneficios de este tipo de terapias. Se trata de una revisión integradora de la literatura basada en la siguiente pregunta orientadora: ¿Es la terapia nutricional enteral beneficiosa para los pacientes sometidos a tratamiento oncológico? La selección de estudios se realizó durante los meses de octubre y noviembre de 2021, en las siguientes bases de datos: Biblioteca Nacional de Medicina (PUBMED), Biblioteca Virtual Elsevier (SCIENCE DIRECT) y Biblioteca Virtual en Salud (BVS) a través del portal Capes Periodical. Inicialmente, la estrategia de búsqueda de los estudios estuvo compuesta por una combinación de descriptores controlados (Terapia nutricional. Nutrición enteral. Beneficios. Pacientes oncológicos) indexados en los Descriptores de Ciencias de la Salud (DeCS / MeSH). Tras el proceso de búsqueda y selección utilizando los criterios mencionados en la metodología, se seleccionaron 7 artículos. Los estudios analizados y discutidos reportan beneficios significativos que realiza la nutrición enteral en pacientes con cáncer, como limitar la pérdida de peso, proporcionar a los pacientes una ingesta energética adecuada, satisfacer las demandas nutricionales aumentadas debido a la enfermedad, mejorar la función inmunológica y gastrointestinal, la recuperación acelerada, la reducción. de complicaciones postoperatorias y tiempo de hospitalización y mejora de indicadores nutricionales.

Palabras clave: Terapia nutricional; Nutrición enteral; Beneficios; Pacientes con cáncer. 
Research, Society and Development, v. 10, n. 17, e234101724196, 2021

(CC BY 4.0) | ISSN 2525-3409 | DOI: http://dx.doi.org/10.33448/rsd-v10i17.24196

\section{Introdução}

O câncer continua sendo uma das principais causas de morte no mundo, e seu percentual vem crescendo nas últimas décadas, atingindo 21,4 milhões de casos e 13,2 milhões de mortes. Embora novas opções terapêuticas estejam sendo pesquisadas em todo o mundo, a maioria das estratégias de tratamento ainda dependem dos tratamentos disponíveis e de outras intervenções que devem melhorar a expectativa de vida dos pacientes em condições curáveis e incuráveis (Co, 2015).

A desnutrição é uma característica comum em pacientes com câncer e é consequência tanto da presença do tumor quanto dos tratamentos médicos e cirúrgicos anticâncer. A desnutrição tem um impacto negativo na qualidade de vida e nas toxicidades do tratamento, e estima-se que até 10-20\% dos pacientes com câncer morrem devido às consequências da desnutrição, e não pelo tumor em si (Muscaritoli et al., 2021).

A desnutrição geralmente ocorre em pacientes com câncer devido ao aumento do metabolismo basal e distúrbios de glicose, bem como metabolismo de gordura e proteína. No geral, o câncer em si consome muitos nutrientes durante o desenvolvimento, resultando em dificuldades alimentares para os pacientes, levando ao aumento da desnutrição devido à quimiorradioterapia e causando sérios danos aos pacientes (Correia et al., 2003; Qiu et al., 2020).

Assim, a nutrição desempenha um papel crucial no tratamento do câncer multimodal. Evidências robustas indicam que as questões nutricionais devem ser levadas em consideração desde o momento do diagnóstico do câncer, dentro de uma via diagnóstica e terapêutica, e devem ocorrer em paralelo com tratamentos antineoplásicos. No entanto, em todo o mundo, a desnutrição relacionada ao câncer ainda é amplamente não reconhecida, subestimada e subtratada na prática clínica (Muscaritoli et al., 2021).

A conduta nutricional assim com a via utilizada dependera da situação clínica do paciente em cuidado. No caso de a nutrição oral permanecer inadequada apesar do aconselhamento nutricional, e na presença de função intestinal normal, a nutrição enteral (NE) total ou suplementar deve ser considerada (Caccialanza et al., 2016).

Ensaios observacionais prospectivos e retrospectivos em pacientes com câncer demonstraram que a NE (melhor se precoce) em comparação com a alimentação oral reduz a perda de peso, a frequência e a duração das interrupções do tratamento e a taxa de internações hospitalares (Lewis et al., 2017).

Diante do exposto, a pesquisa objetiva realizar revisão literária acerca de estudos que aplicaram na prática clínica condutas nutricionais em nutrição enteral para a terapêutica de pacientes com câncer e relatam os benefícios deste tipo de terapia.

\section{Metodologia}

Trata-se de uma revisão integrativa da literatura, conduzida conforme orientações de Souza, Silva e Carvalho (2010). Assim, o estudo seguiu as etapas: elaboração da pergunta norteadora, busca ou amostragem na literatura, coleta de dados, análise crítica dos estudos incluídos, discussão dos resultados e apresentação da revisão integrativa (Souza, silva \& carvalho, 2010). A pergunta norteadora construída foi: A terapia nutricional enteral é benéfica para pacientes em tratamento de câncer?

A seleção dos estudos foi realizada durante os meses de outubro e novembro de 2021, nas bases de dados: National Library of Medicine (PUBMED), Biblioteca virtual da Elsevier (SCIENCE DIRECT) e Biblioteca Virtual em Saúde (BVS) via portal Periódico Capes.

Inicialmente, a estratégia para a busca dos estudos foi composta pela combinação de descritores controlados (Terapia nutricional. Nutrição enteral. Benefícios. Pacientes oncológicos) indexados nos Descritores em Ciências da Saúde (DeCS/MeSH). Realizou-se a combinação dos descritores controlados por intermédio do operador booleano AND como demonstrado na Tabela 1 . 
Tabela 1 - Estratégias de busca utilizadas nas bases Pubmed, Science Direct e Biblioteca virtual em saúde (BVS).

\begin{tabular}{|c|c|c|c|c|}
\hline $\begin{array}{ll}\text { BASE DE } \\
\text { DADOS }\end{array}$ & ESTRATÉGIA DE BUSCA & RESULTADOS & FILTRADOS & SELECIONADOS \\
\hline Pubmed & $\begin{array}{l}\text { Nutritional Therapy and Enteral } \\
\text { Nutrition and Benefits and } \\
\text { Cancer Patients. }\end{array}$ & 11 & 10 & 4 \\
\hline $\begin{array}{l}\text { Science } \\
\text { Direct }\end{array}$ & $\begin{array}{l}\text { Nutritional Therapy and Enteral } \\
\text { Nutrition and Benefits and } \\
\text { Cancer Patients. }\end{array}$ & 20408 & 54 & 2 \\
\hline BVS & $\begin{array}{l}\text { Nutritional Therapy and Enteral } \\
\text { Nutrition and Benefits and } \\
\text { Cancer Patients. }\end{array}$ & 131 & 11 & 1 \\
\hline
\end{tabular}

Fonte: Pesquisa direta (2021).

Além da utilização dos descritores, a seleção dos estudos ocorreu de acordo com os critérios de inclusão que foram: artigos originais completos que realizaram avaliação do impacto da terapia nutricional enteral em pacientes oncológicos. Trabalhos publicados entre os anos de 2016 a 2021 nos idiomas inglês e português, com a presença dos referidos descritores e trabalhos gratuitos disponíveis na integra. Foram excluídos todos os artigos que não corroboravam com o objetivo da pesquisa após leitura dos resumos de cada estudo e estudos realizados com animais.

O levantamento nas bases de dados resultou em 5 artigos em português e 20545 artigos em inglês. Algumas referências foram utilizadas após busca manual dos artigos selecionados. A triagem da busca revelou 18 estudos duplicados, resultando em 20532 registros após remoção das duplicações.

Na primeira triagem, baseada nos critérios de inclusão e exclusão, eliminaram-se 20457 estudos. Após a elegibilidade/avaliação crítica dos textos completos de 75 registros, 7 atenderam aos critérios de inclusão e prosseguiram para fase de extração de dados, leitura exaustiva e síntese do conhecimento.

\section{Resultados e Discussão}

Após o processo de busca e seleção utilizando os critérios citados na metodologia, foram selecionados 7 artigos. $\mathrm{Na}$ tabela 2 é possível visualizar os autores e ano de publicação, título do estudo, metodologia e principais resultados relatados por cada estudo.

Tabela 2. Autores, ano de publicação, título do estudo, metodologia e principais resultados relatados.

\begin{tabular}{|c|c|c|}
\hline Autores/ano/Título & Metodologia & Resultados \\
\hline $\begin{array}{l}\text { Gavazzi et al. (2016). } \\
\text { Impacto da nutrição } \\
\text { enteral domiciliar em } \\
\text { pacientes desnutridos } \\
\text { com câncer }\end{array}$ & $\begin{array}{l}\text { Pacientes com câncer gastrointestinal } \\
\text { superior e candidatos a cirurgia de } \\
\text { grande porte foram incluídos no } \\
\text { protocolo quando a pontuação de } \\
\text { triagem de risco nutricional (NRS 2002). } \\
\text { Todos os pacientes receberam }\end{array}$ & $\begin{array}{l}79 \text { pacientes foram randomizados; } 38 \text { receberam nutrição } \\
\text { enteral continuada em casa e } 41 \text { pacientes receberam apenas } \\
\text { aconselhamento nutricional. Após } 2 \text { meses, os pacientes em } \\
\text { nutrição enteral domiciliar mantiveram seu peso corporal } \\
\text { médio, enquanto os pacientes no grupo de aconselhamento } \\
\text { nutricional apresentaram uma perda de peso de } 3,6 \mathrm{~kg} \text {. }\end{array}$ \\
\hline
\end{tabular}


Research, Society and Development, v. 10, n. 17, e234101724196, 2021

(CC BY 4.0) | ISSN 2525-3409 | DOI: http://dx.doi.org/10.33448/rsd-v10i17.24196

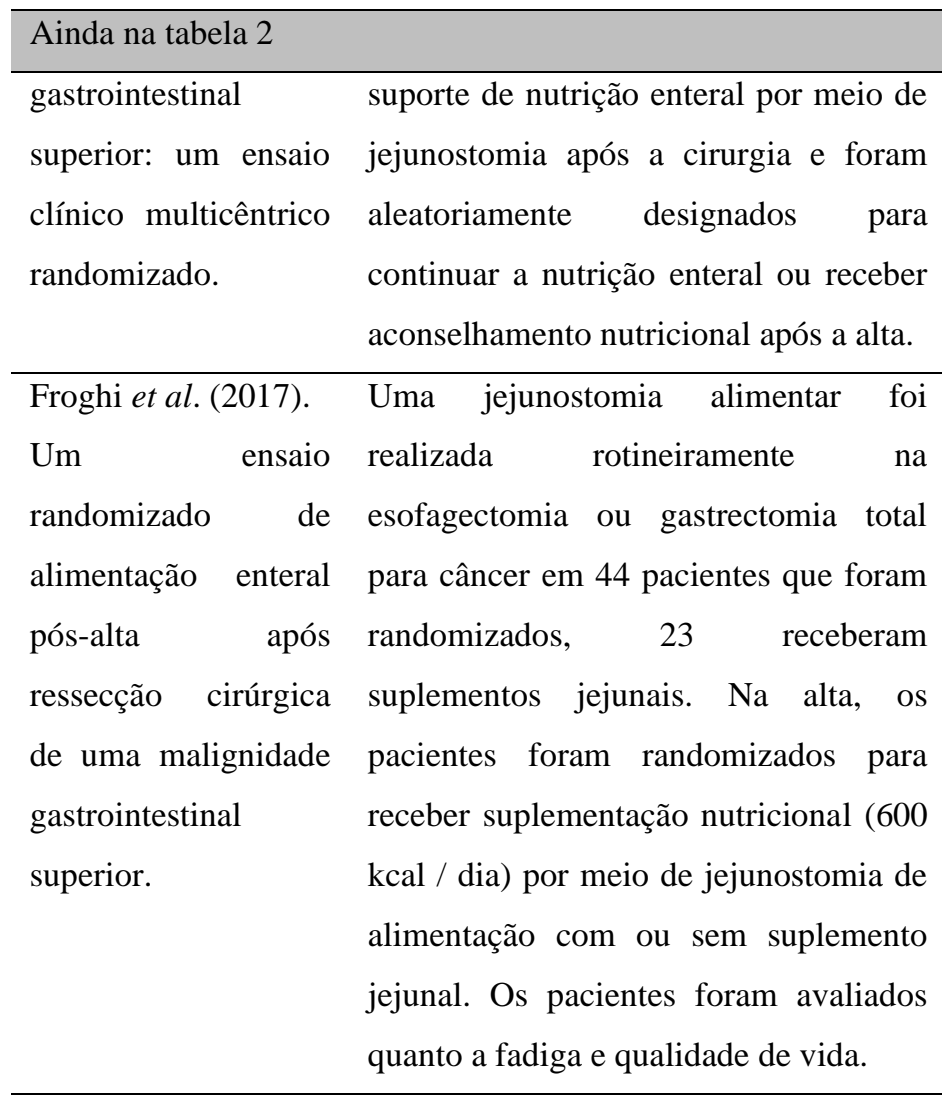

Baker et al. (2017). Um total de 41 participantes com câncer

Ingestão de nutrientes

(32 esofagectomia, 9 gastrectomia total)

6 pacientes que receberam os cuidados habituais necessitaram

e contribuição da

receberam alimentação por jejunostomia

de alimentação de resgate. 6 semanas após a alta hospitalar,

nutrição enteral em casa $(n=18)$ ou cuidados habituais

as ingestões de energia foram atendidas naqueles que

domiciliar

para

sem alimentação com suporte enteral (n

receberam alimentação jejunal por causa da contribuição da

atender

às $=23)$. Na alta hospitalar, a ingestão oral

nutrição enteral. A alimentação jejunal não afetou a ingestão

necessidades

foi adequada para energia e proteína em

nutricionais após $9 \%$ e $6 \%$, respectivamente.

esofagectomia e

gastrectomia total.

oral, sendo semelhante em ambos os grupos (alimentação:

$77 \%$ necessidade estimada, cuidados habituais: $79 \%$ ). Aos 3

meses, a ingestão inadequada de micronutrientes foi

observada em mais de 1/3 dos pacientes. Em comparação com

os valores basais, 6 semanas após a cirurgia foi observada perda de peso superior a 5\% em 5/18 (28\%) dos pacientes que receberam alimentação, 14/17 (82\%) dos que receberam cuidados habituais e 5/6 (83\%) daqueles que necessitaram de alimentação de resgate. A perda de peso foi em média de $4,1 \%$ (no grupo alimentados), $10,4 \%$ (grupo cuidados usuais) e 9,2\% (grupo alimentados com resgate). Essas tendências persistiram por seis meses.

$\begin{array}{ll}\text { Chen } \text { et al. (2017). } & \text { Neste estudo, 68 pacientes confirmados } \\ \text { Os efeitos da nutrição } & \text { com obstrução da saída gástrica em } \\ \text { enteral pré-operatória } & \text { decorrência de câncer gástrico e que } \\ \text { de tubos } & \text { aceitaram realizar a operação foram }\end{array}$
Após pré-NE, os níveis de peso, albumina (ALB), préalbumina e transferrina (TNF) no grupo EN foram significativamente aumentados no dia pré-operação em comparação com o dia de admissão, mas não aumentaram significativamente no grupo NP. O peso dos pacientes no 


\section{Ainda na tabela 2}

de alimentação nasal randomizados em um grupo de NE e um na obstrução da saída gástrica.

grupo de NP. As diferenças no estado nutricional, função imunológica, complicações pós-operatórias, peso dos pacientes, ruídos intestinais e tempo do primeiro flato, tempo do tubo de tração, tempo de internação hospitalar e custo de hospitalização entre o pré e pósoperatório foram todos gravados.

grupo NE aumentaram significativamente em comparação ao grupo NP no dia pré-operatório e no dia da alta; Os níveis de proteína total, ALB, e TNF do grupo EN foram significativamente aumentados em comparação com o grupo PN no pré e pós-operatório dias 1 e 3. Os níveis de CD3 +, CD4 + / CD8 + (linfócitos T), IgA e IgM (imunoglobulinas) no grupo EN foram maiores do que no grupo PN no pré e pósoperatório; o grupo EN teve uma incidência significativamente menor de má cicatrização de feridas, infecção da cavidade peritoneal, pneumonia, e um tempo mais curto do primeiro ruído intestinal, primeiro tempo de flatos e internação pós-operatória do que o grupo PN.

\begin{abstract}
Wu et al. (2018).
142 pacientes desnutridos com câncer de

A nutrição enteral esôfago foram designados para receber domiciliar após esofagectomia minimamente invasiva de esofagectomia Ivor Lewis (grupo MIE) com colocação minimamente invasiva pode melhorar a qualidade de vida e reduzir o risco de desnutrição. de tubo de alimentação jejunal laparoscópica ou esofagectomia aberta (grupo OE) com tubo de alimentação nasojejunal. Foram avaliados a qualidade de vida e o risco de desnutrição.
\end{abstract}

No total, 150 pacientes com câncer de Ding et al. (2020). pulmão foram analisados acelerada combinada com nutrição enteral no tratamento de pacientes com câncer de pulmão. retrospectivamente. 66 pacientes foram divididos aleatoriamente em um grupo controle com nutrição convencional (grupo Con) e um grupo de reabilitação acelerada combinada com nutrição enteral (grupo EN). Foram analisados drenagem pós-operatória; tempo total de internação, albumina, hemoglobina e contagens de linfócitos totais (CPT) antes e depois do tratamento.
67 pacientes foram incluídos no grupo MIE e 75 foram incluídos no grupo OE. Os sintomas relacionados a fadiga, náusea, vômito, dor e perda de apetite diminuíram significativamente nos pacientes tratados com nutrição enteral domiciliar de 3 meses. Da mesma forma, os pacientes tratados com nutrição enteral domiciliar de 3 meses tiveram um risco menor de desnutrição do que os pacientes que não receberam. Mais pacientes no grupo MIE (receberam 3 meses HEN) foram capazes de completar a quimiorradioterapia pósoperatória do que pacientes no grupo OE.

A albumina sérica, pré-albumina e hemoglobina em ambos os grupos diminuíram após a operação e foram significativamente maiores no grupo EN do que no grupo Con. A CPT diminuiu em ambos os grupos após a operação e foi significativamente maior no grupo $\mathrm{EN}$ do que no grupo con. O volume de drenagem pós-operatória, o tempo total de internação foram significativamente menores no grupo NE do que no grupo Con.

\footnotetext{
Wang et al. (2021).

Resultados da

Os sujeitos do estudo foram 227 pacientes que receberam cirurgias de
}

O grupo de nutrição enteral precoce apresentou melhores resultados no tempo até o primeiro flato pós-operatório, o tempo para atingir as metas nutricionais, e a duração da da internação hospitalar em comparação com o grupo de nutrição enteral retardada. Não houve diferenças entre os 
Research, Society and Development, v. 10, n. 17, e234101724196, 2021

(CC BY 4.0) | ISSN 2525-3409 | DOI: http://dx.doi.org/10.33448/rsd-v10i17.24196

\begin{tabular}{ll}
\hline Ainda na tabela 2 & \\
\hline alimentação enteral & ressecção de câncer colorretal. Os grupos nas incidências de vômito, distensão, íleo, vazamento \\
precoce em pacientes & seguintes fatores foram avaliados: risco de anastomose, infecção e interrupções da nutrição enteral. \\
após cirurgia curativa & de desnutrição, duração da anestesia, \\
de câncer colorretal: & nutrição enteral, tempo para a primeiro \\
um & flato pós-operatório, tempo para a \\
comparativo & primeira defecação, sintomas associados \\
retrospectivo. & à alimentação, interrupções nutricionais, \\
& tempo para atingir as metas nutricionais, \\
& complicações pós-operatórias e tempo \\
& de internação hospitalar. Os indivíduos \\
& que receberam nutrição enteral em até \\
& 48 horas após a cirurgia foram \\
& considerados como o grupo de nutrição \\
& enteral precoce.
\end{tabular}

Fonte: Pesquisa direta (2021).

Gavazzi et al. (2016), realizaram estudo com o intuito de avaliar a nutrição enteral domiciliar e aconselhamento na limitação da perda de peso durante o tratamento oncológico. Os autores concluíram em seu estudo que a nutrição enteral domiciliar é um tratamento simples e viável para dar suporte a pacientes desnutridos com câncer gastrointestinal superior, após uma grande cirurgia e durante a quimioterapia, a fim de limitar a perda de peso adicional.

No estudo de Froghi et al. (2017), examinou-se o benefício de uma suplementação nutricional de 6 semanas por meio de uma jejunostomia de alimentação na fadiga, qualidade de vida e vida independente. Os resultados obtidos na realização da pesquisa concluíram que a adição de alimentação jejunal é eficaz em fornecer aos pacientes uma ingestão energética adequada, e o aumento da ingestão de energia, entretanto, não produziu nenhuma melhora significativa nas medidas de fadiga, qualidade de vida ou economia dos gastos hospitalares.

Já Baker et al. (2017) realizaram estudo com a finalidade de avaliar os aspetos nutricionais após ressecção esofagogástrica e a influência da alimentação domiciliar por jejunostomia nos seis meses após a cirurgia. Os autores concluíram ao final do estudo que a alimentação suplementar com jejunostomia contribui de forma importante para atender às demandas nutricionais dos pacientes após a ressecção esofagogástrica. Os autores ainda ressaltam que a ingestão nutricional oral não foi comprometida, afastando a afirmação de que a alimentação jejunal desincentiva os pacientes do ato de comer.

No estudo de Chen et al. (2017) foram examinados pacientes com obstrução da saída gástrica (GOO) que receberam duas semanas de reforço da terapia nutricional enteral pré-operatória (pré-EN) por meio de um tubo de alimentação nasal-jejenal colocado sob um gastroscópio para avaliar a viabilidade e o benefício potencial do pré-NE em comparação com nutrição parenteral (NP). Os autores chegaram à conclusão de que Pré-NE através de um tubo de alimentação nasal-jejuno e colocado sob um gastroscópio em pacientes com obstrução da saída gástrica foi seguro, viável e benéfico para o estado nutricional, função imunológica e função gastrointestinal e recuperação acelerada, embora não aumentasse o custo da hospitalização.

Wu et al. (2018), investigou o efeito da nutrição enteral domiciliar de 3 meses na qualidade de vida relacionada à saúde e no estado nutricional de pacientes com câncer de esôfago que estavam desnutridos no pré-operatório. As conclusões estabelecidas foram que a esofagectomia minimamente invasiva e o tratamento subsequente com nutrição enteral domiciliar de 
Research, Society and Development, v. 10, n. 17, e234101724196, 2021

(CC BY 4.0) | ISSN 2525-3409 | DOI: http://dx.doi.org/10.33448/rsd-v10i17.24196

3 meses podem melhorar a qualidade de vida e reduzir o risco de desnutrição em pacientes desnutridos no pré-operatório.

Na pesquisa de Ding et al. (2020) se investigou o efeito da reabilitação acelerada combinada com nutrição enteral em pacientes com câncer de pulmão tratados cirurgicamente. Os autores concluíram ao final do estudo que o efeito da reabilitação acelerada combinada com nutrição enteral em pacientes com câncer de pulmão é evidente. Os pesquisadores afirmam que a cirurgia leva ao estresse, que aumenta o catabolismo e reduz a síntese de carboidratos, proteínas e gorduras, aumentando o risco nutricional dos pacientes. Ressaltam também que o suporte nutricional combinado com cirurgia torácica minimamente invasiva rápida para pacientes com câncer de pulmão em risco que passam por avaliação e triagem nutricional pré-operatória pode reduzir complicações pós-operatórias e tempo de hospitalização e melhorar os indicadores nutricionais, imunidade, recuperação da função respiratória e resultados clínicos, levando a benefícios socioeconômicos.

Wang et al. (2021), compararam os resultados clínicos de pacientes com e sem nutrição enteral precoce após cirurgia de câncer colorretal. Wang e seus colaboradores concluíram que a alimentação enteral após a cirurgia de câncer colorretal pode acelerar a recuperação das atividades do trato gastrointestinal, promover a ingestão de nutrientes e encurtar o tempo de internação hospitalar sem aumentar as complicações pós-operatórias. Os autores acrescentam que a nutrição enteral é essencial para a recuperação da função gastrointestinal após a cirurgia do câncer colorretal.

\section{Conclusão}

Os estudos analisados e discutidos relatam benefícios significantes desempenhados pela nutrição enteral em pacientes oncológicos, como limitar a perda de peso, fornecer aos pacientes uma ingestão energética adequada, atender às demandas nutricionais aumentadas em decorrência da doença, melhora da função imunológica e função gastrointestinal, recuperação acelerada, redução de complicações pós-operatórias e tempo de hospitalização e melhora dos indicadores nutricionais.

\section{Referências}

Baker, M. L., Halliday, V., Robinson, P., Smith, K., \& Bowrey, D. J. (2017). Nutrient intake and contribution of home enteral nutrition to meeting nutritional requirements after oesophagectomy and total gastrectomy. European journal of clinical nutrition, 71(9), 1121-1128.

Caccialanza, R., Pedrazzoli, P., Cereda, E., Gavazzi, C., Pinto, C., Paccagnella, A., ... \& Zagonel, V. (2016). Nutritional support in cancer patients: a position paper from the Italian Society of Medical Oncology (AIOM) and the Italian Society of Artificial Nutrition and Metabolism (SINPE). Journal of Cancer, 7(2), 131.

Chen, Z. H., Lin, S. Y., Dai, Q. B., Hua, J., \& Chen, S. Q. (2017). The effects of pre-operative enteral nutrition from nasal feeding tubes on gastric outlet obstruction. Nutrients, 9(4), 373.

CO, C. (2015). Cancer Prevention \& Early Detection. Facts \& Figures, 1(1) 10.

Correia, M. I. T., \& Waitzberg, D. L. (2003). The impact of malnutrition on morbidity, mortality, length of hospital stay and costs evaluated through a multivariate model analysis. Clinical nutrition, 22(3), 235-239.

Ding, Q., Chen, W., Gu, Y., Qi, Z. Y., Chen, Y. H., Chen, J., \& Jiang, L. (2020). Accelerated rehabilitation combined with enteral nutrition in the management of lung cancer surgery patients. Asia Pacific Journal of Clinical Nutrition, 29(2), 274-279.

Froghi, F., Sanders, G., Berrisford, R., Wheatley, T., Peyser, P., Rahamim, J., \& Lewis, S. (2017). A randomised trial of post-discharge enteral feeding following surgical resection of an upper gastrointestinal malignancy. Clinical Nutrition, 36(6), 1516-1519.

Gavazzi, C., Colatruglio, S., Valoriani, F., Mazzaferro, V., Sabbatini, A., Biffi, R., ... \& Miceli, R. (2016). Impact of home enteral nutrition in malnourished patients with upper gastrointestinal cancer: a multicentre randomised clinical trial. European Journal of Cancer, 64, $107-112$.

Lewis, S. L., Brody, R., Touger-Decker, R., Parrott, J. S., \& Epstein, J. (2014). Feeding tube use in patients with head and neck cancer. Head \& neck, 36(12), 1789-1795.

Muscaritoli, M., Arends, J., Bachmann, P., Baracos, V., Barthelemy, N., Bertz, H., ... \& Bischoff, S. C. (2021). ESPEN practical guideline: Clinical Nutrition in cancer. Clinical Nutrition, 40(5), 2898-2913.

Qiu, Y., You, J., Wang, K., Cao, Y., Hu, Y., Zhang, H., ... \& Lyu, Q. (2020). Effect of whole-course nutrition management on patients with esophageal cancer undergoing concurrent chemoradiotherapy: A randomized control trial. Nutrition, 69, 110558. 
Research, Society and Development, v. 10, n. 17, e234101724196, 2021

(CC BY 4.0) | ISSN 2525-3409 | DOI: http://dx.doi.org/10.33448/rsd-v10i17.24196

Souza, M. T. D., Silva, M. D. D., \& Carvalho, R. D. (2010). Revisão integrativa: o que é e como fazer. Einstein (São Paulo), 8, $102-106$.

Wang, W. Y., Chen, C. W., Wang, T. J., Lin, K. L., \& Liu, C. Y. (2021). Outcomes of Early Enteral Feeding in Patients after Curative Colorectal Cancer Surgery: A Retrospective Comparative Study. European Journal of Oncology Nursing, 101970.

Wu, Z., Wu, M., Wang, Q., Zhan, T., Wang, L., Pan, S., \& Chen, G. (2018). Home enteral nutrition after minimally invasive esophagectomy can improve quality of life and reduce the risk of malnutrition. Asia Pacific journal of clinical nutrition, 27(1), 129-136. 